\title{
Perfil epidemiológico da sífilis gestacional em uma cidade do nordeste brasileiro: clínica e evolução de 2014 a 2019
}

\author{
Epidemiological profile of gestational syphilis in a city of northeast Brazil: clinical and \\ evolution from 2014 to 2019
}

Perfil epidemiológico de la sífilis gestacional en una ciudad del noreste de Brasil: clínica y evolución de 2014 a 2019

Mickaella Ribeiro da Cunha ${ }^{1 *}$, Andrezza Barbosa Leão ${ }^{1}$, Luciano José Ramos Pimentel Santos ${ }^{1}$, Laércio Pol Fachin¹.

\begin{abstract}
RESUMO
Objetivo: Analisar o perfil epidemiológico de pacientes acometidas por sífilis gestacional no município de Maceió, Alagoas, entre 2014 a 2019. Métodos: Estudo epidemiológico, realizado a partir dos dados do SINAN, disponibilizados pelo DATASUS. Foram analisadas as variáveis: faixa etária, grau de escolaridade, classificação clínica da doença e tratamento. Resultados: No período estudado, foram notificados 1.286 casos de sífilis gestacional no município. Identificou-se um maior crescimento em 2018, com 410 casos notificados. A taxa de incidência anual variou de 27,3 casos/1.000 nascidos vivos em 2018 e 23,3 casos/1.000 nascidos vivos em 2019. Quanto às características maternas a maior prevalência foi na faixa etária de 20 a 29 anos de idade, correspondendo a 662 casos $(51,4 \%)$ e com grau de escolaridade até o fundamental incompleto, correspondente a 541 casos (43\%). Já em relação à classificação clínica da doença a prevalência foi de sífilis primaria com 345 casos $(26,8 \%)$. Conclusão: Evidenciou-se uma aceleração da incidência de casos no período analisado, com predominância de SG primária, com prevalência nas mulheres com idade entre 20 e 29 anos, e de menor escolaridade.
\end{abstract}

Palavras-chave: Doenças sexualmente transmissíveis, Gestante, Sífilis, Transmissão vertical de doença infecciosa, Vigilância epidemiológica.

\begin{abstract}
Objective: To analyze the epidemiological profile of patients affected by gestational syphilis in the city of Maceió, Alagoas, between 2014 and 2019. Methods: Epidemiological study, carried out using SINAN data, made available by DATASUS. The variables were analyzed: age group, education level, clinical classification of the disease and treatment. Results: During the study period, 1,286 cases of gestational syphilis were reported in the municipality. Further growth was identified in 2018, with 410 reported cases. The annual incidence rate ranged from 27.3 cases / 1,000 live births in 2018 and 23.3 cases / 1,000 live births in 2019. As for maternal characteristics, the highest prevalence was in the age group of 20 to 29 years old, corresponding to 662 cases $(51.4 \%)$ and with an education level up to incomplete primary education, corresponding to 541 cases (43\%). Regarding the clinical classification of the disease, the prevalence was primary syphilis with 345 cases $(26.8 \%)$. Conclusion: There was an acceleration of the incidence of cases in the analyzed period, with a predominance of primary SG, with prevalence in women aged between 20 and 29 years, and with less education.
\end{abstract}

Keywords: Sexually transmitted diseases, Pregnant woman, Syphilis, Infectious disease transmission vertical, Epidemiological surveillance.

\section{RESUMEN}

Objetivo: Analizar el perfil epidemiológico de pacientes afectadas por sífilis gestacional en la ciudad de Maceió, Alagoas, entre 2014 y 2019. Métodos: Estudio epidemiológico, a partir de datos del SINAN, puestos a disposición por DATASUS. Se analizaron las variables: grupo de edad, nivel educativo, clasificación clínica de la enfermedad y tratamiento. Resultados: Durante el período de estudio se

${ }^{1}$ Centro Universitário CESMAC, Maceió - AL. *E-mail: mickaellaribeiro@hotmai.com 
reportaron 1.286 casos de sífilis gestacional en el municipio. Se identificó un mayor crecimiento en 2018, con 410 casos notificados. La tasa de incidencia anual osciló entre 27,3 casos / 1.000 nacidos vivos en 2018 y 23,3 casos / 1.000 nacidos vivos en 2019. En cuanto a las características maternas, la mayor prevalencia se dio en el grupo de edad de 20 a 29 años, correspondiente a 662 casos $(51,4 \%$ ) y con un nivel educativo hasta primaria incompleta, correspondiente a 541 casos (43\%). En cuanto a la clasificación clínica de la enfermedad, la prevalencia fue la sífilis primaria con 345 casos $(26,8 \%)$. Conclusión: Hubo una aceleración de la incidencia de casos en el período analizado, con predominio de SG primaria, con prevalencia en mujeres entre 20 y 29 años, y con menor nivel educativo.

Palabras clave: Enfermedades de transmisión sexual, Embarazada, Sífilis, Transmisión vertical de enfermedades infecciosa, Vigilancia epidemiológica.

\section{INTRODUÇÃO}

As infecções sexualmente transmissíveis (IST) são consideradas um problema de saúde pública e estão entre as patologias transmissíveis mais comuns, afetando a saúde e a vida das pessoas em todo o mundo. As IST têm impacto direto sobre a saúde reprodutiva e infantil, à medida que acarretam infertilidade e complicações na gravidez e no parto, além de causar morte fetal e agravos à saúde da mãe e do feto. Elas também têm um impacto indireto na facilitação da transmissão sexual do vírus da imunodeficiência humana (HIV), sendo esse um risco 18 vezes maior que na população não acometida (BRASIL, 2020).

A sífilis é uma infecção sistêmica, de evolução progressiva quando não tratada adequadamente. É causada pela bactéria espiroqueta Treponema pallidum e apresenta-se como uma doença variável, com diversas características clínicas e diferentes estágios (sífilis primária, secundária, latente e terciária). Entidade conhecida desde o século XV, seu estudo ocupa a totalidade das especialidades médicas. É identificada nas formas adquirida e congênita, sendo esta última de notificação compulsória desde 1986 e a gestacional, desde 2005. A principal via de transmissão é o contato sexual (sífilis adquirida), seguido pela transmissão vertical para o feto através da placenta durante gestação de uma mãe com sífilis não tratada de maneira eficaz. Pode ser transmitida ainda por transfusão sanguínea, sendo essa a forma menos comum nos dias atuais (BRASIL, 2017).

A inclusão da sífilis gestacional (SG) como IST de notificação compulsória se justifica pela sua alta prevalência e taxa de transmissão vertical que varia entre $30 \%$ a $100 \%$ se não tratada ou tratada inadequadamente (MARQUES JVS, et al. 2018). No Brasil, o número de casos notificados de sífilis na gestação tem aumentado a cada ano, especialmente após a ausência da penicilina no país durante os anos de 2014 a 2016, o que deu lugar a falta de tratamento dos pacientes portadores da doença, facilitando assim a transmissão (VÁSQUES GGH, 2018).

O diagnóstico e tratamento de SG são de extrema importância para a interrupção da transmissão da doença ao feto e/ou recém-nascido, já que, uma vez que a gestante é tratada adequadamente, as chances de transmissão vertical diminuem consideravelmente, fato que comprova a necessidade de estudo da epidemiologia da doença. Sabendo disso, a subnotificação de casos da SG pode apresentar-se como fator de agravo da doença, sendo assim, um pré-natal de qualidade deve notificar e tratar as gestantes de forma adequada para reduzir os índices de sífilis congênita (SC) (MAGALHÃES SOBRINHO DDT, 2017).

De acordo com o Ministério da Saúde (MS, no Brasil 50 mil puérperas são diagnosticadas com Sífilis ao ano, com prevalência variando de 1,1 a $11,5 \%$, devido à assistência pré-natal e alfabetização maternal (LAFETA KRG, et al., 2016). É uma doença por vezes assintomática que persiste sendo um problema de saúde pública (LIMA TM, et al., 2019). Diante disso, foi implantada a Rede Cegonha que tem como objetivo melhorar a qualidade da assistência prestada à mulher e à criança com foco na atenção ao parto, ao nascimento, ao crescimento e desenvolvimento da criança do zero aos vinte e quatro meses como também garantir acesso ao acolhimento e resolutividade na Rede de Atenção à Saúde Materna e Infantil, reduzindo a mortalidade materna e infantil (BRASIL, 2011).

Dados epidemiológicos recentes revelam um aumento de 300\% do número de casos da SG no Brasil entre os anos de 2010 e 2016. Isso se deve ao aumento do número de testagens e a elevação no número 
de notificações via Sistema de Informação de Agravos de Notificação (Sinan), portanto, sabe-se que ainda existe um grande percentual de casos não notificados, cenário esse que pode ser mais grave do que os dados indicam (BRASIL, 2020).

No Brasil, em 2019 foram notificados 61.127 casos de Sífilis em gestantes (taxa de detecção de 72,8 casos/100.000 nascidos vivos), desses 13.026 estão no Nordeste ( $21,3 \%)$, sendo assim, pelo segundo ano consecutivo, a segunda região brasileira com mais casos de SG notificados, ficando atrás apenas da região Sudeste (BRASIL, 2020).

O estado de Alagoas vem apresentando variação significativa no número de casos da SG nos últimos anos, passou-se de 3 casos notificados em 2010 para 201 casos em 2019. Vale salientar que, no ano de 2018, a capital Maceió esteve entre as capitais com taxa de detecção superior a taxa nacional. Deve-se atentar ainda para possíveis subnotificações nesse período, fato que provoca interferência nas políticas públicas de saúde (BRASIL, 2020).

Diante disso, a perspectiva é que a doença permanecerá em um patamar semelhante ou mais grave nos próximos anos, exigindo assim um novo olhar para esse problema de saúde pública. Com o panorama da SG agravada, avaliar o perfil epidemiológico das pacientes infectadas proporciona a elaboração de estratégias de saúde que promovam a intervenção para diagnóstico e tratamento precoces da doença (BRASIL, 2020).

Considerando o crescente aumento de notificações de SG e SC é de fundamental importância conhecer o perfil das mulheres grávidas e crianças infectadas com sífilis, bem como identificar as áreas com maior foco para esses agravos, para que se possa adotar medidas de redução dessas infecções e propor medidas de intervenção efetivas (BRASIL, 2017).

Visto isso, este artigo tem como objetivo avaliar o perfil epidemiológico de pacientes acometidas por SG em Maceió - AL entre o período de 2014 a 2019. A sua importância se deve ao fato de que a avaliação dos dados disponibilizados pelo Departamento de Informática do Sistema Único de Saúde (DATASUS) pode auxiliar tanto no manejo do problema como no desenvolvimento de políticas públicas de saúde que tenham o propósito de melhorias nas ações relacionadas à prevenção, a assistência pré-natal, o tratamento adequado da sífilis e consequentemente diminuir a incidência de casos de Sífilis/ano no município de Maceió.

\section{MÉTODOS}

Trata-se de um estudo epidemiológico descritivo, cujos dados foram obtidos a partir da consulta na base de dados Sinan disponibilizados pelo DATASUS. Os dados são derivados das notificações compulsórias dos casos de sífilis em gestantes identificados nos residentes de Maceió, no período de 1 de janeiro de 2014 a 31 de dezembro de 2019.

As definições dos casos de sífilis em gestantes são preconizadas pelo Ministério da Saúde e apresentadas nas fichas de notificação/investigação do Sinan. No caso das gestantes, essas são notificadas durante o pré-natal quando apresentam evidência clínica e/ou sorologias positivas. Neste estudo, foram incluídos todos os dados de notificação de SG pertencentes ao munícipio de Maceió registradas no período.

Localizado na região Nordeste, Maceió é a capital do estado de Alagoas, sendo o munícipio mais populoso do estado. A cidade tem uma população estimada em 1.025 .360 milhões de habitantes, com densidade demográfica de $1.854,10$ habitante por quilômetro ${ }^{2}$, vive um intenso crescimento econômico e de infraestrutura. Seu território apresenta 148 pontos de atendimento do Sistema Único de Saúde (SUS) e apresenta-se como centro de referência em saúde para diversas cidades vizinhas (IBGE, 2019).

As seguintes características epidemiológicas foram adotadas na análise do perfil das gestantes incluídas no estudo, foram analisadas as variáveis referentes às características sociodemográficas (faixa etária e escolaridade), bem como a classificação clínica e terapêutica da doença. 
A tabulação dos dados foi realizada no programa Microsoft Excel, onde as variáveis foram organizadas por ano, de modo a compreender o perfil temporal das infecções e o processo saúde-doença no munícipio. Junto a isso, o programa Microsoft Power Point foi utilizado para a elaboração dos gráficos que ilustram a discussão deste artigo.

Por utilizar dados do Sinan, plataforma online do Ministério da Saúde com dados disponíveis em caráter de "domínio público", este estudo não necessitou de aprovação por parte de um Comitê de Ética em Pesquisa com seres humanos, em conformidade com a Resolução ํo 510/2016 do Comitê Nacional em Saúde.

\section{RESULTADOS E DISCUSSÃO}

Conforme os dados expostos no Sinan, Maceió apresentou um total de 1.286 casos de SG notificados no período de 2014 a 2019. Observou-se 78 casos em 2014 e 2015 (6,06\%), 121 casos em 2016 (9,4\%) e 249 casos em 2017 (19,36\%). O ápice foi atingido em 2018 com notificação de 410 casos (31,88\%) e segue com 350 casos em $2019(27,21 \%)^{1}$, sendo que a quantidade de casos em 2018 foi significativamente maior do que em 2019 ( $p=0,009)$ (Figura 1) (BRASIL, 2020)

De acordo com esses dados, observou-se em 2019 uma redução de 3,3\% na taxa de detecção de sífilis em gestante em comparação ao ano de 2018. Embora exista a diminuição dos casos de sífilis notificados em quase todo o país, vale salientar que parte dessa redução pode estar relacionada à identificação de problemas na transferência de dados entre as esferas de gestão do SUS, o que pode gerar a diferença no total de casos entre as bases de dados das esferas de governo (municipal, estadual e federal). O declínio nesses números pode também ser resultado de uma demora na notificação de alimentação das bases de dados devido à mobilização dos profissionais de saúde causada de pela atual pandemia de COVID-19 (BRASIL, 2020).

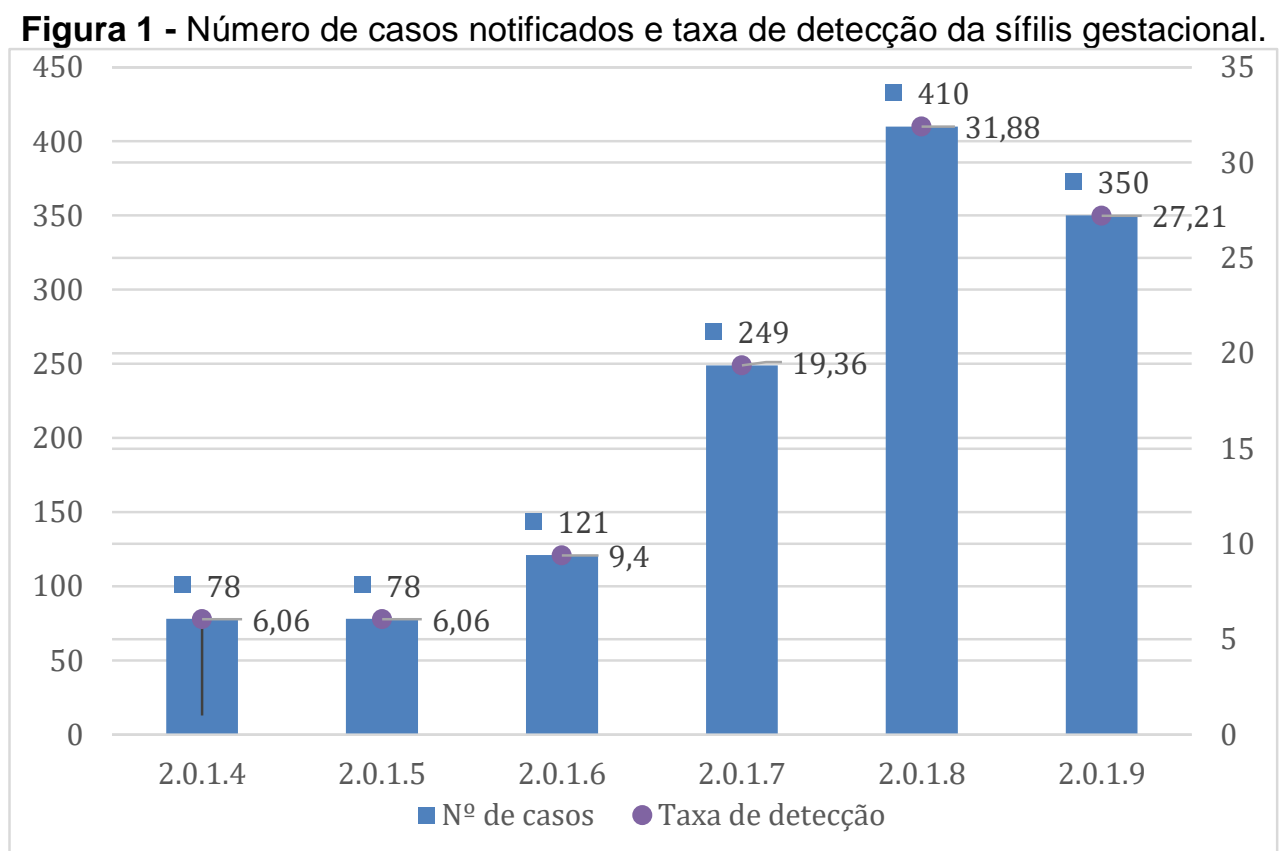

Fonte: Cunha MR, et al., 2020, dados extraídos do SINAN.

Uma das hipóteses desse aumento de casos está relacionada à ampliação da utilização de testes rápidos nos rastreios em gestantes e a uma mudança comportamental em relação à diminuição do uso de preservativos, como indicam dados do MS. Dessa forma, o alto número de casos demonstra que as atuais medidas de prevenção têm sidos ineficazes e acreditam-se que os números possam ser maiores devido à subnotificação de casos. Situações como essas são descritas em outros estudos, a exemplo de um estudo realizado entre 2012 e 2017 na cidade de Sobral - CE, munícipio da região nordeste (MARQUES JVS, et al., 2018). 
É possível ainda que o aumento da incidência seja explicado pelas situações de vulnerabilidade que englobam desde as características comportamentais e reprodutivas às práticas sexuais inseguras ${ }^{20}$. É reconhecido que a adoção de comportamentos sexuais seguros é um fator de estudo complexo, não dependente apenas do nível educacional, renda ou acesso a informações, abrange também fatores de significados atribuídos à sexualidade individual. Além disso, o preservativo ainda é visto por muitos como símbolo de infidelidade ou desconfiança, sendo resguardado seu uso apenas em parceiros "desconhecidos" (RIBEIRO KC, et al. 2011).

Junto às medidas ineficazes de promoção e prevenção, existiu um fato no Brasil que dificultou 0 tratamento dos pacientes, facilitando assim a transmissão, principalmente por contato sexual e vertical. A falta da penicilina entre os anos de 2014 e 2016, droga de escolha para terapêutica eficaz da doença, deu lugar ao não tratamento e consequente aumento dos casos em todo o Brasil. Os dados nacionais descrevem a falta do medicamento em $60 \%$ dos estados durante o período descrito acima. Esses dados ainda indicam um total de 65.000 casos de sífilis adquirida em 2015, sendo destes 33.000 casos de SG (VASQUEZ GGH, 2018).

Em relação à faixa etária das gestantes portadoras da sífilis, os dados do período do estudo mostram que houve 21 casos entre 10 e 14 anos ( $1,63 \%$ do total), 368 casos entre 15 e 19 anos (28,61\%), 662 casos entre 20 e 29 anos (51,47\%), 209 casos entre 30 e 39 anos (16,25\%) e 26 casos em mulheres maiores de 40 anos (2,02\%) (BRASIL, 2020). Tais dados reforçam a importância da necessidade de políticas públicas voltadas à prevenção, rastreio e notificação principalmente entre as mulheres jovens em idade fértil, e apontam uma quantidade estatisticamente maior de gestantes na faixa de 20-29 anos como portadoras da doença ( $p<0,0001$, teste de qui-quadrado) (Tabela 1 ).

Tabela 1 - Faixa etária dos casos de sífilis gestacional. Maceió, 2014-2019.

\begin{tabular}{cccccc}
\hline \multicolumn{6}{c}{ FAIXA ETÁRIA } \\
\hline ANO & $\mathbf{1 0 - 1 4}$ anos & $\mathbf{1 5 - 1 9}$ anos & $\mathbf{2 0 - 2 9}$ anos & $\mathbf{3 0 - 3 9}$ anos & $\mathbf{4 0}$ anos ou mais \\
\hline 2014 & 2 & 25 & 33 & 17 & 1 \\
2015 & 4 & 24 & 37 & 10 & 3 \\
2016 & 2 & 48 & 55 & 14 & 2 \\
2017 & 5 & 60 & 142 & 39 & 3 \\
2018 & 6 & 110 & 207 & 76 & 11 \\
2019 & 2 & 101 & 188 & 53 & 6 \\
Total & $\mathbf{2 1}$ & $\mathbf{3 6 8}$ & $\mathbf{6 6 2}$ & $\mathbf{2 0 9}$ & $\mathbf{2 6}$ \\
\hline
\end{tabular}

Fonte: Cunha MR, et al., 2020, dados extraídos do SINAN.

Um estudo realizado no Brasil entre 2011 e 2012 envolvendo 23.894 mulheres das cinco regiões do país evidencia resultados próximos aos encontrados em Maceió no que se relaciona a faixa etária das mulheres acometidas pela Sífilis. Entre as 16.807 mulheres portadoras da SG, 70,3\% estavam com idade entre 20-34 anos, fato esse que se repete no município em estudo (DOMINGUE RMSM, et al., 2014).

Os números de casos entre as jovens de 15 e 19 anos são preocupantes. Um estudo realizado reforça a importância de ação entre essa população, o estudo afirma que esta faixa etária de mulheres apresenta maior chance de início de pré-natal tardio, levando ao atraso do diagnóstico e tratamento, isso aumenta 0 risco de transmissão e complicações fetais (JEZO RFV, et al., 2017).

Em relação à escolaridade das gestantes, a SG acomete com maior frequência a população com Ensino Fundamental incompleto, sendo 531 casos, o que representa aproximadamente $43 \%$ do total. Incluem-se nesse grupo: analfabetas e pessoas que não finalizaram o $9^{\circ}$ ano (antiga $8^{a}$ série fundamental). Esse grupo representa aproximadamente 50\% do total de notificações de SG entre 2014 e 2019. Esses dados podem ser ainda maiores considerando que 330 casos $(25,6 \%)$ tiveram o grau de escolaridade ignorado durante a notificação (Figura 2) (BRASIL, 2020). 
A SG está presente em maiores números em mulheres com baixa escolaridade, o que parece estar relacionado à dificuldade de obtenção de informações sobre formas a doença, como formas de prevenção, diagnóstico e tratamento. Outros estudos corroboram esta ideia, como é o caso de resultados demonstrados em um estudo realizado na cidade de Sobral - CE, que avaliou o maior número de casos em mulheres com menor grau de escolaridade (MARQUES JVS, et al., 2018). Outro estudo realizado no Rio de Janeiro avaliou dados das cinco regiões do país e evidenciou um valor 3,2 vezes maior de casos de SG entre mulheres com até 7 anos de estudo em comparação às com maior acesso a escolaridade (DOMINGUES RMSM, et al., 2014)

Outra análise epidemiológica realizada no Tocantins também obteve resultados semelhantes no que se refere à escolaridade. Esse estudo indica que $75 \%$ das mulheres acometidas pela SG variavam o grau de estudo entre Ensino Fundamental incompleto e Ensino Médio completo (CAVALCANTE PAM, et al. 2017). Esses fatos são capazes de identificar uma população de risco para a doença e a grande relevância das ações de conscientização e ações de prevenção e conhecimento da patologia.

Figura 2 - Casos de sífilis gestacional por escolaridade.

Fonte: Cunha MR, et al., 2020, dados extraídos do SINAN. 
A classificação clínica da SG mostra uma predominância da sífilis primária com 345 casos $(26,8 \%$ do total). Um dado que chama a atenção é quantidade de notificações classificadas como ignoradas, fato que poderia mudar o cenário da classificação da SG no município, este representa $32,5 \%$ dos casos (418), liderando os números na classificação clínica da doença (Tabela 2) (BRASIL, 2020).

Tabela 2 - Casos de sífilis gestacional por classificação clínica da doença.

\begin{tabular}{ccc}
\hline CLASSIFICAÇÃO CLÍNICA & $\mathbf{N}$ & $\%$ \\
\hline PRIMÁRIA & 345 & $26,8 \%$ \\
SECUNDÁRIA & 146 & $11,35 \%$ \\
TERCIÁRIA & 71 & $5,52 \%$ \\
LATENTE & 306 & $23,8 \%$ \\
IGNORADA & 418 & $32,5 \%$ \\
TOTAL & 1286 & $100 \%$ \\
\hline
\end{tabular}

Fonte: Cunha MR, et al., 2020, dados extraídos do SINAN.

A classificação da doença está diretamente ligada aos testes diagnósticos utilizados. No Brasil, o teste não treponêmico VDRL é o método de teste amplamente disponível para triagem, seguimento terapêutico e controle de cura. A confirmação laboratorial com testes treponêmicos como TPHA e FTA-Abs estão disponíveis também na rede SUS, porém sua ausência não deve retardar a conduta da doença, visto que o tratamento precoce é de grande valia nos resultados (MAGALHÃES DMS, et al., 2013).

Sobre a classificação clínica da doença, temos a forma primária como mais prevalente no município, porém, os casos identificados como ignorados tomam conta do maior valor. Esse fato dificulta a interpretação do momento de diagnóstico das pacientes. A relevância desse dado diz respeito ao risco de transmissão vertical da doença, que é maior nas fases primária e secundária. Sabe-se que a caracterização da sífilis é fundamental para a adequação terapêutica (BRASIL, 2017).

A distribuição dos casos em relação ao tratamento do período de 2016 e 2019 um total de 1.130 casos. Observa-se maior prevalência, em comparação com qualquer outro $(p<0,0001)$, entre as mulheres que realizaram com o tratamento com a Penicilina - primeira linha de tratamento (956 casos) representando $84,60 \%$ do total. As mulheres que não realizaram nenhum tipo de tratamento somam 41 casos $(3,62 \%)$, e as que realizaram outro esquema de tratamento foram 17 casos (1,50\%). Os casos ignorados foram 116 casos $(10,26 \%)$ (Figura 3) (BRASIL, 2020).

Figura 3 - Casos de sífilis gestacional de acordo com o tratamento.

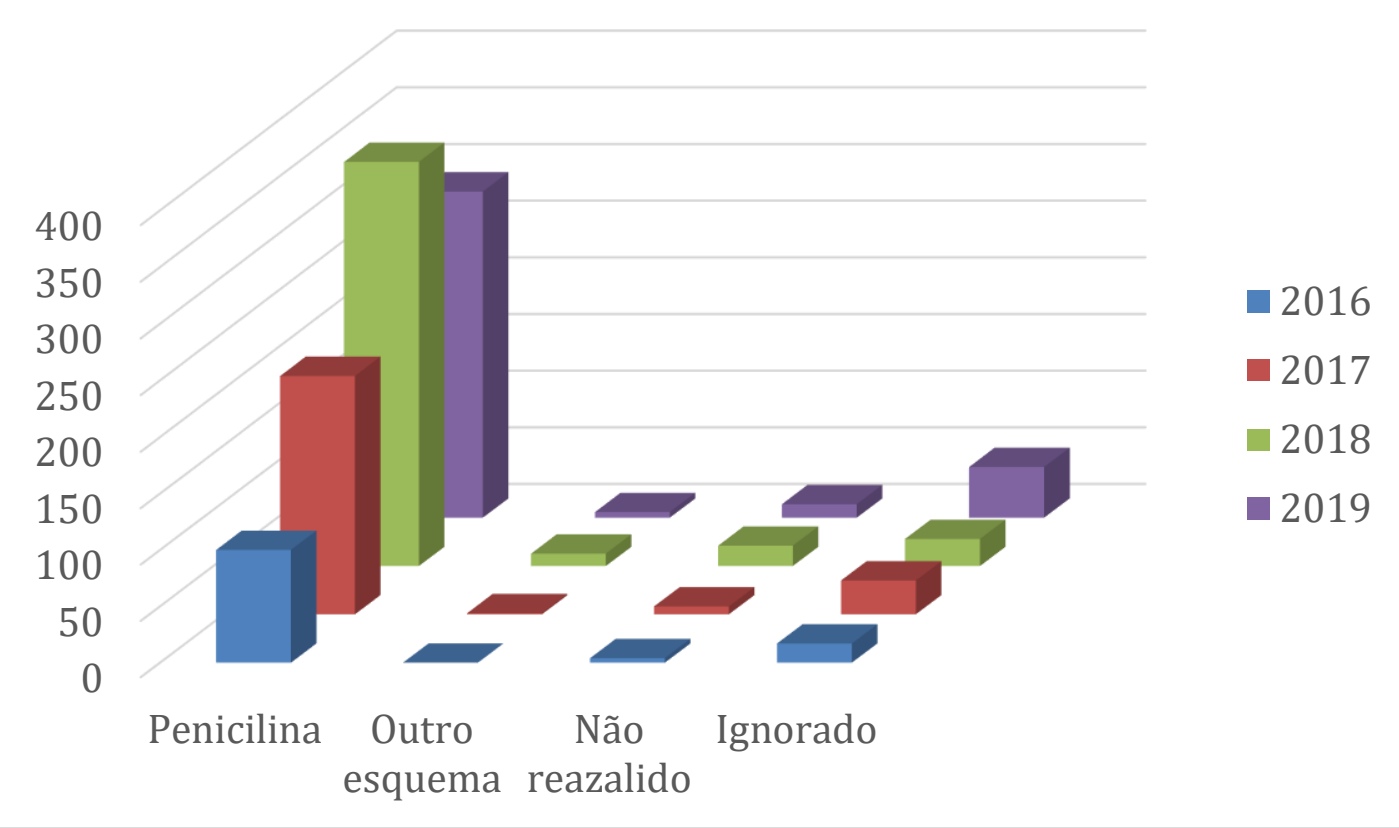

Fonte: Cunha MR, et al., 2020 , dados extraídos do SINAN. 
A escolha do tratamento da SG é feita a partir da sua classificação sendo utilizada como primeira linha de tratamento a Penicilina, sendo essa a única opção segura e eficaz para o tratamento das gestantes. Para sífilis primária, secundária ou latente recente administra-se Penicilina Benzatina 2,4 milhões UI, IM, dose única, sendo 1,2 milhão UI em casa glúteo. Na Sífilis terciária, latente tardia ou de duração ignorada, administra-se Penicilina Benzatina 2,4 milhões UI, IM, 1x/semana, por três semanas (PCDT, 2020).

Um estudo realizado em São Paulo mostra que as mulheres com baixa frequência às consultas de prénatal apresentam maior risco para SG, efeito que diminui à medida que cresce o número de consultas. Embora esses achados sejam esperados, eles levantam aspectos que precisam ser levados em conta por todos os mediadores da assistência pré-natal, é preciso ações de aconselhamento, vigilância epidemiológica e o rastreamento de parceiros, já que esses comprometem o controle da transmissão da doença e se apresentam como um dos fatores de falha de tratamento (MACÊDO VC, et al., 2017).

É necessário incorporar ações de vigilência epidemiológica com o objetivo de aconselhamento com informações relevantes, confiáveis e atualizadas. De tal maneira, alguns indicadores de vigilância epidemiológica irão seguir na avaliação com revisão de prontuário, notificação de sífilis congênita, notificação de gestantes com sífilis, rastreamento de parceiros e o aconselhamento sobre a necessidade da triagem para sífilis (MACEDO VC, et al., 2009).

As evidências das desigualdades sociais em saúde no país oferecem base para a hipótese de que a SG está relacionada ao baixo nível socioeconômico e a dificuldade ao acesso de serviços de saúde e educação. Isso contribui para a persistência da transmissão vertical e expõe as fragilidades de acesso ao sistema de saúde, oportunidade de rastreamento, diagnóstico e tratamento das gestantes e parceiros (MAGALHÃES DMS, et al., 2013).

O Ministério da Saúde lançou em 2000 o Programa de Humanização do Pré-Natal e Nascimento, com o objetivo de garantir a ampliação do acesso, cobertura e assistência pré-natal, parto e puerpério. O programa estabelece critérios para realização de um acompanhamento adequado como mínimo de seis consultas prénatal, triagem sorológica com teste VDRL na primeira consulta e trigésima semana de gestação, testagem anti-HIV na primeira consulta e aplicação de vacina antitetânica. Esses aspectos objetivam a redução da morbimortalidade materna e perinatal, à medida que permitem $\mathrm{O}$ adequado diagnóstico, tratamento e seguimento das pacientes (MAGALHÃES DMS, et al., 2013).

\section{CONCLUSÃO}

Os resultados do presente estudo evidenciam aumento na quantidade de casos da SG em relação aos números notificados em anos anteriores. Evidenciou-se predominância de SG primária, com prevalência nas mulheres com idade entre 20 e 29 anos, e de menor escolaridade. Identifica-se que as condições de vulnerabilidade, sejam elas comportamentais, de acesso à educação ou de acesso à assistência de saúde estão significativamente associadas à Sífilis em gestantes. Com isso, apesar da doença apresentar métodos diagnósticos e tratamento simples, continua como um grave problema de saúde e social. A sífilis se apresenta como um grande problema de saúde pública no Brasil, passível de tratamento e prevenção. No entanto, é visto que a falha da abordagem preventiva, diagnóstica e da intervenção durante o pré-natal nas gestantes portadoras eleva o risco complicações. Visto isso, fica clara a necessidade de intervenções mais potentes no que se refere a políticas públicas voltadas à prevenção, diagnóstico e tratamento precoces da doença.

\section{REFERÊNCIAS}

1. BRASIL. 2011. Em: Gabinete do Ministro. Portaria no 1.459. Disponível em: http://www.as.saude.ms.gov.br/wpcontent/uploads/2016/07/Portaria-GM-MS-n\%C2\%BA1.459-do-dia-24-de-junho-de-2011.pdf. Acesso em: 8 nov 2020.

2. BRASIL. 2017. Em: Boletim epidemiológico de sífilis. Disponível em: http://www.aids.gov.br/pt-br/pub/2017/boletimepidemiologico-de-sifilis-2017. Acesso em: 7 nov 2020.

3. BRASIL. 2017. Em: Guia de vigilância em saúde. Disponível em: http://bvsms.saude.gov.br/bvs/publicacoes/guia_vigilancia_saude_volume_2.pdf. Acesso em: 7 nov 2020. 
4. BRASIL. 2020. Em: Boletim epidemiológico: sífilis 2020. Disponível em: http://www.aids.gov.br/ptbr/pub/2020/boletim-sifilis-2020. Acesso em: 7 Nov 2020.

5. CAVALCANTE PAM, et al. Sífilis gestacional e congênita em Palmas, Tocantins, 2017-2014. Epidemiol Serv Saúde., 2017; 26: 255-64.

6. CRUZEIRO ALS, et al. Comportamento sexual de risco: fatores associados ao número de parceiros sexuais e ao uso de preservativos em adolescentes. Ciênc. Saúde Coletiva. 2010; 15: 1149-58.

7. DOMINGUES RMSM, et al. Prevalence of syphilis in pregnancy and prenatal syphilis testing in Brazil: birth in Brazil study. Ver Saúde Pública, 2014; 48: 766-74.

8. IBGE. 2019. Cidades: Maceió. Disponível em: https://cidades.ibge.gov.br/brasil/al/maceio/panorama. Acesso em: 6 nov 2020.

9. JEZO RFV, et al. Gravidez na Adolescência: Perfil das Gestantes e Mães Adolescentes em uma Unidade Básica de Saúde. Revista de Enfermagem do Centro-Oeste Mineiro, 2017; 1387: 1-8.

10. LAFETA KRG, et al. Sífilis materna e congênita, subnotificação e difícil controle. Rev. bras. Epidemiol., 2016; $19: 63-$ 74.

11. LIMA TM, et al. Perfil epidemiológico de pacientes com sífilis congênita e gestacional em um município do Estado de São Paulo, Brasil. Rev. Bras. Saúde Mater. Infant., 2019; 19: 873-880.

12. MACEDO VC, et al. Risk factors for syphilis in women: case-control study. Rev. saúde pública. 2017; 510:78.

13. MACEDO VC, et al. Avaliação das ações de prevenção da transmissão vertical do HIV e sífilis em maternidades públicas de quatro municípios do nordeste brasileiro. Cad. Saúde Pública. 2009; 25: 679-92.

14. MAGALHÃES DMS, et al. Sífilis materna e congênita: ainda um desafio. Cad Saude Publica. 2013; $29: 1109-20$.

15. MAGALHÃES SOBRINHO DDT. Sífilis gestacional: investigação da fragilidade do tratamento na Estratégia Saúde da Família. Programa de pós-graduação em ciências médicas, 2017.

16. MARQUES JVS, et al. Perfil epidemiológico da sífilis gestacional: clínica e evolução de 2012 a 2017. Sanare, 2018; 17: $13-20$.

17. MELO NGDO, et al. Diferenciais intraurbanos de sífilis congênita no Recife, Pernambuco, Brasil (2004-2006). Epidemiol. Serv. Saúde. 2011; 20: 213-22.

18. Protocolo Clínico e Diretrizes Terapêuticas para prevenção da transmissão vertical do HIV, Sífilis e Hepatites Virais. 2020. Disponível

em: http://conitec.gov.br/images/Consultas/Relatorios/2020/PCDT_PTV_HIV_CP_42_2020.pdfAcesso em: 6 nov 2020.

19. RIBEIRO KCS, et al. Querer é poder? A ausência do uso do preservativo nos relatos de mulheres jovens. DST-J Bras. Doenç. Sex Transm. 2011; 23: 84-9.

20. VAZQUEZ GGH. Vênus nos braços de mercúrio, bismuto e arsênio Notas históricas sobre sífilis gestacional antes da penicilina. Sexualidad, Salud y Sociedad, 2018; 226-245. 